minerals revealed signatures of chloropyromorphite after one month of incubation. The mineral became the dominant transformation product after three months. Lead not exposed to fungi showed typical signs of corrosion.

This is the first time that microbes have been found to generate pyromorphite. Curr. Biol. http://dx.doi. org/10.1016/j.cub.2011.12.017 (2012)

\section{Plant gobbles buried worms}

The Brazilian plant Philcoxia minensis feasts on nematode worms - making this spindly plant the first known to trap its prey using sticky leaves buried underground.

Philcoxia (pictured) grows in the white, nutrient-poor sands of the Brazilian Cerrado, a biodiversity hot spot. Rafael Oliveira at the State University of Campinas in São Paulo, Brazil, and his colleagues found dead nematodes trapped on $P$. minensis's subterranean leaves. The team fed the plants nematodes containing labelled nitrogen. After two days, about $15 \%$ of the prey's labelled nitrogen was found in the leaves.

This, as well as the presence of enzymes called phosphatases on the leaves, suggests that the plant actively digests its prey.

Proc. Natl Acad. Sci. USA http://dx.doi.org/10.1073/ pnas.1114199109 (2012)

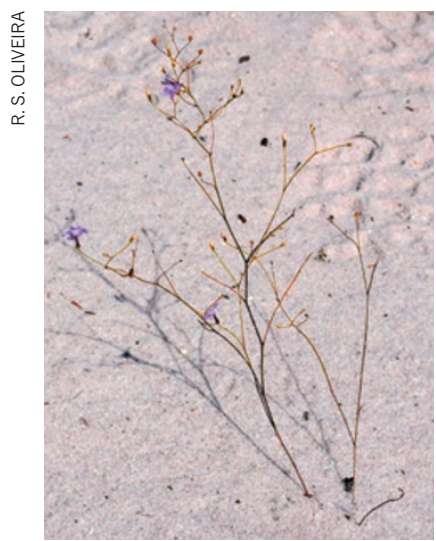

\section{How parasites get sticky}

To invade host cells, one group of parasites relies on a protein that promotes the release of sticky molecules from a specialized organelle called a microneme.

Parasites of the phylum Apicomplexa, which includes those responsible for malaria (Plasmodium falciparum) and toxoplasmosis (Toxoplasma gondii), secrete a mixture of adhesion proteins at their surfaces, allowing them to stick to host cells. Marc-Jan Gubbels at Boston College in Massachusetts and his colleagues sequenced the genome of a mutant strain of Toxoplasma with an impaired ability to release its microneme contents or invade cells. They found a mutation in a protein called DOC2.1. Shutting off the gene encoding an equivalent protein in Plasmodium also impaired this parasite's ability to invade. Science 335, 218-221 (2012)

\section{ATMOSPHERIC SCIENCE}

\section{More aerosols, more rain}

Atmospheric aerosols seem to boost the intensity of rainfall over large areas of land and ocean.

Aerosols - tiny particles that include black carbon and sulphates - influence cloud properties, but whether the overall effect produces more or less rain has been uncertain. Ilan Koren at the Weizmann Institute of Science in Rehovot, Israel, and his colleagues looked at satellite observations of rainfall, aerosols and clouds between June and August 2007, and compared them with meteorological data. They found that increases in aerosol abundance correlated with a higher rate of rainfall in the tropics, subtropics and mid-latitudes.

The authors say that the similar trends seen across different locations and

COMMUNITY CHOICE

The most viewed papers in science

DEVELOPMENTAL BIOLOGY

\title{
Watching cells die in real time
}

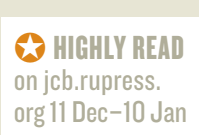

A type of programmed cell death called apoptosis has been imaged in live mouse embryos, providing insight into a developmental process that can go awry and cause neural birth defects.

Yoshifumi Yamaguchi and Masayuki Miura at the University of Tokyo and their team engineered mice that generate a fluorescent signal in response to the activation of caspases, enzymes that trigger apoptosis. The authors used a fastscanning confocal microscope to detect this activation in developing mouse embryos at single-cell resolution. They also conducted time-lapse imaging of an early-developmental process called neural-tube closure - in which apoptosis occurs and the basic structure of the central nervous system is formed.

In embryos that were missing key apoptotic genes, this closure process occurred much more slowly and, in some embryos, even failed to complete. The authors suggest that neural-tube closure must occur within a specific time window to be completed properly.

J. Cell Biol. 195, 1047-1060 (2011)

environmental conditions suggest a link between increased aerosol levels and more intense rainfall.

Nature Geosci. http://dx.doi. org/10.1038/ngeo1364 (2012)

\section{APPLIED PHYSICS}

\section{Magnetic switch for memory}

Some data-storage devices rely on ferromagnets - in which the neighbouring magnetic spins of atoms are aligned - to hold information as magnetic bits. However, magnetic fields can corrupt stored data. Antiferromagnets, in which the neighbouring spins are oppositely aligned, are not as sensitive to magnetic fields, so are a promising alternative. Sebastian Loth, Andreas Heinrich and their colleagues at IBM's Almaden Research Center in San Jose, California, have read and switched the magnetic states of antiferromagnetic iron atoms, demonstrating that information can be stored antiferromagnetically.

The authors placed a few

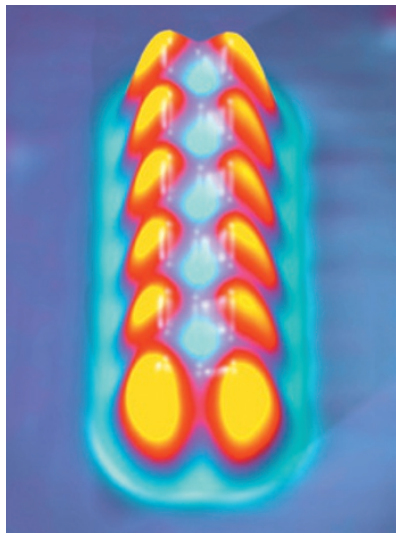

antiferromagnetic iron atoms in a regular pattern (pictured) on a copper nitride surface, and switched their magnetic states by applying a current of more than 7 millivolts to individual atoms using the tip of a scanning tunnelling microscope. From images taken with the microscope, the authors could distinguish two stable and distinct magnetic states of the atoms.

Science 335, 196-199 (2012)

\section{$\rightarrow$ NATURE,COM}

For the latest research published by Naturevisit:

www.nature.com/latestresearch 